\title{
The spectral problem and *-representations of algebras associated with Dynkin graphs.
}

\author{
STANISLAV KRUGLJAK, STANISLAV POPOVYCH, YURII SAMOILENKO *
}

4th June 2018

\begin{abstract}
We study the connection between *-representations of algebras associated with graphs, locally-scalar graph representations and the problem about the spectrum of a sum of two Hermitian operators. For algebras associated with Dynkin graphs we give an explicit description of the parameters for which there are irreducible representations and an algorithm for contructing these representations.
\end{abstract}

KEYWORDS: Hilbert space, irreducible representation, graph, quiver, Coxeter functor, Horn's problem, Dynkin diagram, *-algebra

AMS SUBJECT CLASSIFICATION: 16W10, 16G20, 47L30

\section{Introduction.}

1. Let $A, B, C$ be Hermitian $n \times n$ matrices with given eigenvalues, $\tau(A)=$ $\left\{\lambda_{1}(A) \geq \lambda_{2}(A) \geq \ldots \geq \lambda_{n}(A)\right\}, \tau(B)=\left\{\lambda_{1}(B) \geq \lambda_{2}(B) \geq \ldots \geq \lambda_{n}(B)\right\}, \tau(C)=$ $\left\{\lambda_{1}(C) \geq \lambda_{2}(C) \geq \ldots \geq \lambda_{n}(C)\right\}$. The well-known classical problem about spectrum of a sum of two Hermitian matrices (Horn's problem) is to describe a connection between $\tau(A), \tau(B), \tau(C)$ for matrices $A, B, C$ such that $A+B=C$.

A recent solution of this problem (see. [3, 4] and others) gives a complete description of possible $\tau(A), \tau(B), \tau(C)$ in terms of linear inequalities of the form

$$
\sum_{i \in I} \lambda_{i}(A)+\sum_{j \in J} \lambda_{j}(B) \geq \sum_{k \in K} \lambda_{k}(C)
$$

where $I, J, K$ are certain subsets of $\{1, \ldots, n\}$. Note that the number of necessary inequalities increases with $n$.

2. Consider the following modifications of the problem mentioned above, called henceforth the spectral problem (res. strict spectral problem). We will consider bounded linear Hermitian operators on a separable Hilbert space $H$. For an operator $X$ denote

${ }^{*}$ Insitute of Mathematics of National Academy of Sciences, Kyiv, Ukraine 
by $\sigma(X)$ its spectrum. Let $M_{1}, M_{2}, M_{3}$ be given closed subsets of $\mathbb{R}^{+}$and $\gamma \in \mathbb{R}^{+}$. The problem consists of the following: 1)to determine whether there are Hermitian operators $A, B, C$ on $H$ such that $\sigma(A) \subseteq M_{1}, \sigma(B) \subseteq M_{2}, \sigma(C) \subseteq M_{3}$ (res. $\sigma(A)=M_{1}$, $\left.\sigma(B)=M_{2}, \sigma(C)=M_{3}\right)$ and $A+B+C=\gamma I$ ? 2) if the answer is in the affirmative, to give a description (up to unitary equivalence) of the operators. In this work the sets $M_{1}, M_{2}, M_{3}$ will be finite. Note that even for finite $M_{k}$ the second part of the problem can be very complicated if $\left|M_{k}\right|$ is large enough.

The essential difference with the classical Horn's problem is that we do not fix the dimension of $H$ (it may be finite or infinite) and the spectral multiplicities. It seems that solution of spectral and strict spectral problems could not be deduced directly from inequalities of the form (0.1), since the number of necessary inequalities increases with $n$.

3. These problems could be reformulated in terms of ${ }^{*}$-representations of ${ }^{*}$-algebras. Namely, let $\alpha=\left(\alpha_{1}, \alpha_{2}, \ldots, \alpha_{k}\right), \beta=\left(\beta_{1}, \beta_{2}, \ldots, \beta_{l}\right), \delta=\left(\delta_{1}, \delta_{2}, \ldots, \delta_{m}\right)$ be vectors with positive strictly decreasing coefficients. Let us remark that we can assume that each set $M_{1}, M_{2}, M_{3}$ contains zero (this can be achieved by a translation). Henceforth, $M_{1}=\alpha \cup\{0\}, M_{2}=\beta \cup\{0\}, M_{3}=\delta \cup\{0\}$. Let us consider the associative algebra defined by the following generators and relations (see. [7]):

$$
\begin{gathered}
\mathcal{P}_{\alpha, \beta, \delta, \gamma}=\mathbb{C}\left\langle p_{1}, p_{2}, \ldots, p_{k}, q_{1}, q_{2}, \ldots, q_{l}, s_{1}, s_{2}, \ldots, s_{m}\right| \\
p_{i} p_{j}=\delta_{i j} p_{i}, q_{i} q_{j}=\delta_{i j} q_{i} ; s_{i} s_{j}=\delta_{i j} s_{i} \\
\left.\sum_{i=1}^{k} \alpha_{i} p_{i}+\sum_{j=1}^{l} \beta_{j} q_{j}+\sum_{d=1}^{m} \delta_{d} s_{d}=\gamma e\right\rangle .
\end{gathered}
$$

Here $e$ is the identity of the algebra, $\delta_{i j}$ is the Kronecker symbol. This is a *-algebra, if we declare all generators to be self-adjoint.

A $*$-representation $\pi$ of $\mathcal{P}_{\alpha, \beta, \delta, \gamma}$ is determined by a triple of non-negative operators $A=\sum_{i=1}^{k} \alpha_{i} P_{i}, B=\sum_{j=1}^{l} \beta_{j} Q_{j}, C=\sum_{d=1}^{m} \delta_{d} S_{d}$, where each of the families of orthoprojections, $\left\{I-\sum_{i=1}^{k} P_{i}, P_{i}=\pi\left(p_{i}\right), i=1, \ldots, k\right\},\left\{I-\sum_{j=1}^{l} Q_{j}\right.$, and $Q_{j}=$ $\left.\pi\left(q_{j}\right), j=1, \ldots, l\right\}$ and $\left\{I-\sum_{d=1}^{m} S_{d}, S_{d}=\pi\left(s_{d}\right), d=1, \ldots, m\right\}$, forms a resolution of the identity and such that $A+B+C=\gamma I$. So in terms of $*$-representations, the spectral problem is a problem consisting of the following two parts: 1) a description of the set $\Sigma_{k, l, m}$ of the parameters $\alpha, \beta, \delta, \gamma$ for which there exist $*$-representations of $\left.\mathcal{P}_{\alpha, \beta, \delta, \gamma} ; 2\right)$ a description of ${ }^{*}$-representations $\pi$ of the ${ }^{*}$-algebra $\mathcal{P}_{\alpha, \beta, \delta, \gamma}$. A natural way to try to solve the spectral problem is to describe all irreducible $*$-representations up to unitary equivalence and then all *-representations as sums or direct integrals of irreducible representations.

An essential step in this direction is to describe of irreducible non-degenerate representations. Let us introduce non-degenerate representations and then reformulate the strict spectral problem in terms of ${ }^{*}$-representations of algebras. Let us call a *-representation $\pi$ of the algebra $\mathcal{P}_{\alpha, \beta, \delta, \gamma}$ non-degenerate, if $\pi\left(p_{i}\right) \neq 0, \pi\left(q_{j}\right) \neq 0$, $\pi\left(s_{d}\right) \neq 0$ for $1 \leq i \leq k, 1 \leq j \leq l, 1 \leq d \leq m$, and $\sum_{i=1}^{k} \pi\left(p_{i}\right) \neq I, \sum_{j=1}^{l} \pi\left(q_{j}\right) \neq I$, $\sum_{d=1}^{m} \pi\left(s_{d}\right) \neq I$. Consider the following sets: $T_{k, l, m}=\left\{(\alpha, \beta, \delta, \gamma) \mid \alpha \in \mathbb{R}^{+k}, \beta \in\right.$ $\left.\mathbb{R}^{+l}, \delta \in \mathbb{R}^{+m}, \gamma \in \mathbb{R}^{+}\right\}$and $W=\left\{(\alpha, \beta, \delta, \gamma) \in T_{k, l, m} \mid\right.$ there is a non-degenerate ${ }^{*}$-representation of $\left.\mathcal{P}_{\alpha, \beta, \delta, \gamma}\right\}$; they depend only on $(k, l, m)$. With an integer vector 
$(k, l, m)$ we will associate non-oriented star-shape graph $G$ with three branches of the lengths $k, l$ and $m$ stemming from single root. Henceforth we will denote $W$ by $W(G)$, and $T_{k, l, m}$ by $T(G)$, where $G$ is the tree mentioned above. We will need some more notations $W_{i r r}=\{(\alpha, \beta, \delta, \gamma) \in T(G) \mid$ there exists a non-degenerate irreducible ${ }^{*}$-representation of $\left.\mathcal{P}_{\alpha, \beta, \delta, \gamma}\right\}$. The strict spectral problem for operators on a Hilbert space can be reformulated in the following way: 1) for a given graph $G$ describe the set $W(G) ; 2$ ) describe non-degenerate representations $\mathcal{P}_{\alpha, \beta, \delta, \gamma}$ up to unitary equivalence. 4. A common part of the spectral and strict spectral problems is a description of the set $W_{i r r}(G)$ and irreducible representations. The present article is devoted to this common part for Dynkin graphs.

If the graph is a Dynkin graph, the problem is greatly simplified. The algebras $\mathcal{P}_{\alpha, \beta, \delta, \gamma}$ associated with Dynkin graphs (res. extended Dynkin graphs) have a more simple structure then in other cases. In particular, the algebras $\mathcal{P}_{\alpha, \beta, \delta, \gamma}$ are finite dimensional, (res. have polynomial growth) if and only if the associated graph is a Dynkin graph (res. an extended Dynkin graph) (see. 8]). As shown in [6] irreducible representations of the algebras associated with Dynkin graphs exist only in certain dimensions that are bounded from above. In the paper we give a complete description of $W(G)_{i r r}$ for all Dynkin graphs $G$ and an algorithm for finding all irreducible representations.

In Sec. 1 and Sec. 2 we will show that the theory of $*$-representations of the algebras associated with such graphs can be reformulated in terms of locally-scalar graph representations.

In Sec. 3 we summarize the calculation of possible parameters and generalized dimensions of non-degenerate irreducible $*$-representations of the $*$-algebras associated with Dynkin graphs using the machinery elaborated in [6].

Since the description of $*$-representations of the $*$-algebras associated with Dynkin graphs can be reduced to a description of non-degenerate irreducible representations (see [9]), in this article we essentially solve the spectral problem for the algebras associated with Dynkin graphs.

The results of the paper were partially announced in $[5$.

Let us remark that representations of quivers were also studied in connection with Horn's problem in [2].

\section{Locally-scalar graph representations and rep- resentations of the algebras generated by ortho- projections.}

Henceforth we will use definitions, notations and results about representations of graphs in category of Hilbert spaces found in [6]. Let us recall some of them.

A graph $G$ consists of a set of vertices $G_{v}$ a set of edges $G_{e}$ and a map $\varepsilon$ from $G_{e}$ into the set of one- and two-element subsets of $G_{v}$ (the edge is mapped into the set of incident vertices). Henceforth we consider connected finite graphs without

cycles (trees). Fix a decomposition of $G_{v}$ of the form $G_{v}=\stackrel{\circ}{G}_{v} \sqcup \stackrel{\bullet}{G}_{v}$ (unique up 
to permutation) such that for each $\alpha \in G_{e}$ one of the vertices from $\varepsilon(\alpha)$ belongs to $\stackrel{\circ}{G}_{v}$ and the other to $\stackrel{\bullet}{G}_{v}$. Vertices in $\stackrel{\circ}{G}_{v}$ will be called even, and those in the set $\dot{G}_{v}$ odd. Let us recall the definition of a representation $\Pi$ of a graph $G$ in the category of Hilbert spaces $\mathcal{H}$. Let us associate with each vertex $g \in G_{v}$ a Hilbert space $\Pi(g)=H_{g} \in \mathrm{ObH}$, and with each edge $\gamma \in G_{e}$ such that $\varepsilon(\gamma)=\left\{g_{1}, g_{2}\right\}$ a pair of mutually adjoint operators $\Pi(\gamma)=\left\{\Gamma_{g_{1}, g_{2}}, \Gamma_{g_{2}, g_{1}}\right\}$, where $\Gamma_{g_{1}, g_{2}}: H_{g_{2}} \rightarrow H_{g_{1}}$. Construct a category $\operatorname{Rep}(G, \mathcal{H})$, its objects are representations of the graph $G$ in $\mathcal{H}$. A morphism $C: \Pi \rightarrow \widetilde{\Pi}$ is a family $\left\{C_{g}\right\}_{g \in G_{v}}$ of operators $C_{g}: \Pi(g) \rightarrow \widetilde{\Pi}(g)$ such that the following diagrams commute for all edges $\gamma_{g_{2}, g_{1}} \in G_{e}$ :

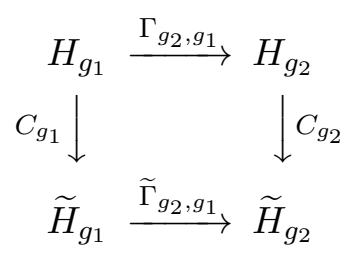

Let $M_{g}$ be the set of vertices connected with $g$ by an edge. Let us define the operators

$$
A_{g}=\sum_{g^{\prime} \in M_{g}} \Gamma_{g g^{\prime}} \Gamma_{g^{\prime} g}
$$

A representation $\Pi$ in $\operatorname{Rep}(G, \mathcal{H})$ will be called locally-scalar, if all operators $A_{g}$ are scalar, $A_{g}=\alpha_{g} I_{H_{g}}$. The full subcategory $\operatorname{Rep}(G, \mathcal{H})$, objects of which are locallyscalar representations, will be denoted by $\operatorname{Rep} G$ and called the category of locallyscalar representations of the graph $G$.

Let us denote by $V_{G}$ the real vector space consisting of sets $x=\left(x_{g}\right)$ of real numbers $x_{g}, g \in G_{v}$. Elements $x$ of $V_{G}$ we will call $G$-vectors. A vector $x=\left(x_{g}\right)$ is called positive, $x>0$, if $x \neq 0$ and $x_{g} \geq 0$ for all $g \in G_{v}$. Denote $V_{G}^{+}=\{x \in$ $\left.V_{G} \mid x>0\right\}$. If $\Pi$ is a finite dimensional representation of the graph $G$ then the $G$ vector $(d(g))$, where $d(g)=\operatorname{dim} \Pi(g)$ is called the dimension of $\Pi$. If $A_{g}=f(g) I_{H_{g}}$ then the $G$-vector $f=(f(g))$ is called a character of a locally-scalar representation $\Pi$ and $\Pi$ is called an $f$-representation in this case. The support $G_{v}^{\Pi}$ of $\Pi$ is $\{g \in$ $\left.G_{v} \mid \Pi(g) \neq 0\right\}$. A representation $\Pi$ is faithful if $G_{v}^{\Pi}=G_{v}$. A character of the locallyscalar representation $\Pi$ is uniquely defined on the support $G_{v}^{\Pi}$ and non-uniquely on its complement. In the general case, denote by $\left\{f_{\Pi}\right\}$ the set of characters of $\Pi$. For each vertex $g \in G_{v}$, denote by $\sigma_{g}$ the linear operator on $V_{G}$ given by the formulae:

$$
\begin{gathered}
\left(\sigma_{g} x\right)_{g^{\prime}}=x_{g^{\prime}} \text { if } g^{\prime} \neq g, \\
\left(\sigma_{g} x\right)_{g}=-x_{g}+\sum_{g^{\prime} \in M_{g}} x_{g^{\prime}} .
\end{gathered}
$$

The mapping $\sigma_{g}$ is called a reflection at the vertex $g$. The composition of all reflections at odd vertices is denoted by $\dot{c}$ (it does not depend on the order of the factors), and at all even vertices by $\stackrel{\circ}{c}$. A Coxeter transformation is $c=\stackrel{\circ}{c} \cdot, c^{-1}=\stackrel{\bullet}{c} c$. The transformation $\dot{c}(\stackrel{c}{c})$ is called an odd (even) Coxeter map. Let us adopt the following notations for compositions of the Coxeter maps: $\stackrel{\bullet}{c}_{k}=\ldots \stackrel{\bullet \bullet c c}{c}(k$-factors $), \stackrel{\circ}{c_{k}}=\ldots \stackrel{\bullet}{ } \cdot \stackrel{\circ}{c}$ ( $k$ - factors), $k \in \mathbb{N}$. 
Any real function $f$ on $G_{v}$ can be identified with a $G$-vector $f=(f(g))_{g \in G_{v}}$. If $d(g)$ is the dimension of a locally-scalar graph representation $\Pi$, then

$$
\begin{aligned}
& \stackrel{\circ}{c}(d)(g)= \begin{cases}-d(g)+\sum_{g^{\prime} \in M_{g}} d\left(g^{\prime}\right), & \text { if } g \in \stackrel{\circ}{G}_{v}, \\
d(g), & \text { if } g \in \dot{G}_{v},\end{cases} \\
& \stackrel{\bullet}{c}(d)(g)= \begin{cases}-d(g)+\sum_{g^{\prime} \in M_{g}} d\left(g^{\prime}\right), & \text { if } g \in \dot{G}_{v}, \\
d(g), & \text { if } g \in \stackrel{\circ}{G}_{v} .\end{cases}
\end{aligned}
$$

For $d \in Z_{G}^{+}$and $f \in V_{G}^{+}$, consider the full subcategory $\operatorname{Rep}(G, d, f)$ in $\operatorname{Rep} G$ (here $Z_{G}^{+}$is the set of positive integer $G$-vectors), with the set of objects $O b \operatorname{Rep}(G, d, f)=$ $\left\{\Pi \mid \operatorname{dim} \Pi(g)=d(g), f \in\left\{f_{\Pi}\right\}\right\}$. All representations $\Pi$ from $\operatorname{Rep}(G, d, f)$ have the same support $X=X_{d}=G_{v}^{\Pi}=\left\{g \in G_{v} \mid d(g) \neq 0\right\}$. We will consider these categories only if $(d, f) \in S=\left\{(d, f) \in Z_{G}^{+} \times V_{G}^{+} \mid d(g)+f(g)>0, g \in G_{v}\right\}$. Let $\stackrel{\circ}{X}=X \cap \stackrel{\circ}{G}_{v}$, $\dot{X}=X \cap \dot{G}_{v} . \operatorname{Rep}_{\circ}(G, d, f) \subset \operatorname{Rep}(G, d, f)\left(\operatorname{Rep}_{\bullet}(G, d, f) \subset \operatorname{Rep}(G, d, f)\right)$ is the full subcategory with objects $(\Pi, f)$ where $f(g)>0$ if $g \in \stackrel{\circ}{X}(f(g)>0$ if $g \in \dot{X})$. Let $S_{0}=\left\{(d, f) \in S \mid f(g)>0\right.$ if $\left.g \in \stackrel{\circ}{X}_{d}\right\}, S_{\bullet}=\left\{(d, f) \in S \mid f(g)>0\right.$ if $\left.g \in \dot{X}_{d}\right\}$

Put

$$
\begin{aligned}
& \dot{c}_{d}(f)(g)=\stackrel{\circ}{f}_{d}(g)= \begin{cases}\stackrel{\bullet}{c}(f)(g), & \text { if } g \in \dot{\bullet}_{d}, \\
f(g), & \text { if } g \notin \dot{\bullet}_{d},\end{cases} \\
& \stackrel{\circ}{c}_{d}(f)(g)=\stackrel{\circ}{f}_{d}(g)= \begin{cases}\circ \\
c(f)(g), & \text { if } g \in \stackrel{\circ}{X}_{d}, \\
f(g), & \text { if } g \notin \stackrel{\circ}{X}_{d} .\end{cases}
\end{aligned}
$$

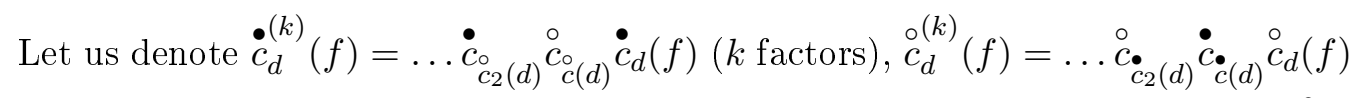
( $k$ factors). The even and odd Coxeter reflection functors are defined in $[\underline{6}, \stackrel{\circ}{F}$ : $\operatorname{Rep}_{\circ}(G, d, f) \rightarrow \operatorname{Rep}_{\circ}\left(G, \stackrel{\circ}{c}(d), \stackrel{\circ}{f_{d}}\right)$ if $(d, f) \in S_{\circ}, \stackrel{\bullet}{F}: \operatorname{Rep}_{\bullet}(G, d, f) \rightarrow \operatorname{Rep}_{\bullet}\left(G, \stackrel{\bullet}{c}(d), \stackrel{\bullet}{f}_{d}\right)$ if $(d, f) \in S_{\bullet}$; they are equivalences of the categories. Let us denote $\stackrel{\circ}{F}_{k}(\Pi)=$ $\ldots \stackrel{\circ}{F} \stackrel{\circ}{F} \stackrel{\circ}{F}(\Pi)$ ( $k$ factors), $\stackrel{\circ}{F}_{k}(\Pi)=\ldots \stackrel{\circ}{F} \stackrel{\circ}{F} \dot{F}(\Pi)$ ( $k$ factors), if the compositions exist. Using these functors, an analog of Gabriel's theorem for graphs and their locallyscalar representations has been proven in [6]. In particular, it has been proved that any locally-scalar graph representation decomposes into a direct sum (finite or infinite) of finite dimensional indecomposable representations, and all indecomposable representations can be obtained by odd and even Coxeter reflection functors starting from the simplest representations $\Pi_{g}$ of the graph $G\left(\Pi_{g}(g)=\mathbb{C}, \Pi_{g}\left(g^{\prime}\right)=0\right.$ if $\left.g \neq g^{\prime} ; g, g^{\prime} \in G_{v}\right)$. 


\section{Representations of algebras generated by pro- jections.}

Let us consider a tree $G$ with vertices $\left\{g_{i}, i=0, \ldots, k+l+m\right\}$ and edges $\gamma_{g_{i} g_{j}}$, see the figure.

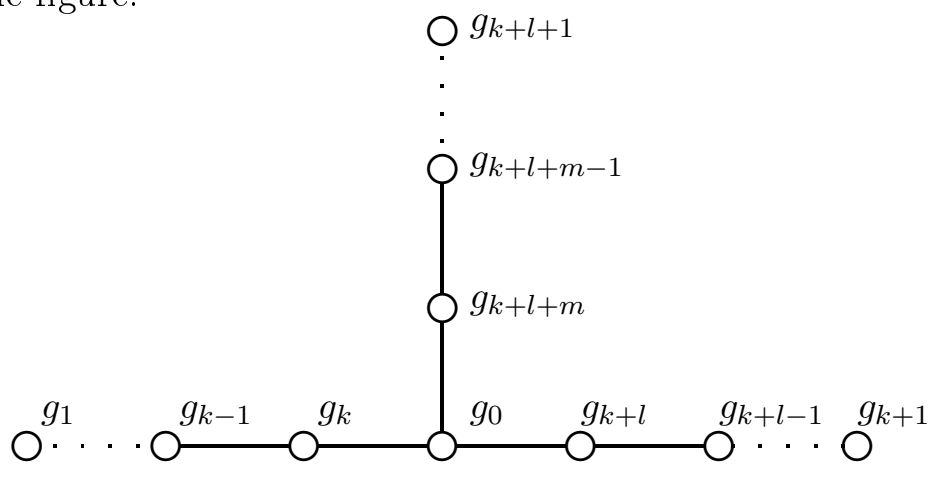

We will establish a connection between $*$-representations of the $*$-algebra $\mathcal{P}_{\alpha, \beta, \delta, \gamma}$ and locally-scalar representations of the graph $G$, see [5], $\alpha=\left(\alpha_{1}, \alpha_{2}, \ldots, \alpha_{k}\right), \beta=$ $\left(\beta_{1}, \beta_{2}, \ldots, \beta_{l}\right), \delta=\left(\delta_{1}, \delta_{2}, \ldots, \delta_{m}\right)$.

Definition 1 An irreducible finite dimensional *-representation $\pi$ of the algebra $\mathcal{P}_{\alpha, \beta, \delta, \gamma}$ such that $\pi\left(p_{i}\right) \neq 0(1 \leq i \leq k), \pi\left(q_{j}\right) \neq 0(1 \leq j \leq l), \pi\left(s_{d}\right) \neq 0(1 \leq d \leq m)$, and $\sum_{i=1}^{k} \pi\left(p_{i}\right) \neq I, \sum_{j=1}^{l} \pi\left(q_{j}\right) \neq I, \sum_{d=1}^{m} \pi\left(s_{d}\right) \neq I$, will be called non-degenerate. $B y \overline{\operatorname{Rep}} \mathcal{P}_{\alpha, \beta, \delta, \gamma}$ we will denote the full subcategory of non-degenerate representations in the category $\operatorname{Rep} \mathcal{P}_{\alpha, \beta, \delta, \gamma}$ of $*$-representations of the $*$-algebra $\mathcal{P}_{\alpha, \beta, \delta, \gamma}$ in the category $\mathcal{H}$ of Hilbert spaces.

Let $\pi$ be a $*$-representation of $\mathcal{P}_{\alpha, \beta, \delta, \gamma}$ on a Hilbert space $H_{0}$. Put $P_{i}=\pi\left(p_{i}\right)$, $1 \leq i \leq k, Q_{j}=\pi\left(q_{j}\right), 1 \leq j \leq l, S_{t}=\pi\left(s_{t}\right), 1 \leq t \leq m$. Let $H_{p_{i}}=\Im \mathrm{m} P_{i}$, $H_{q_{j}}=\Im \mathrm{m} Q_{j}, H_{s_{t}}=\Im \mathrm{m} S_{t}$. Denote by $\Gamma_{p_{i}}, \Gamma_{q_{j}}, \Gamma_{s_{t}}$ the corresponding natural isometries $H_{p_{i}} \rightarrow H_{0}, H_{q_{j}} \rightarrow H_{0}, H_{s t} \rightarrow H_{0}$. Then, in particular, $\Gamma_{p_{i}}^{*} \Gamma_{p_{i}}=I_{H_{p_{i}}}$ is the identity operator on $H_{p_{i}}$ and $\Gamma_{p_{i}} \Gamma_{p_{i}}^{*}=P_{i}$. Similar equalities hold for the operators $\Gamma_{q_{i}}$ and $\Gamma_{s_{i}}$. Using $\pi$ we construct a locally-scalar representation $\Pi$ of the graph $G$.

Let $\Gamma_{i j}: H_{j} \rightarrow H_{i}$ denote the operator adjoint to $\Gamma_{j i}: H_{i} \rightarrow H_{j}$, i.e. $\Gamma_{i j}=\Gamma_{j i}^{*}$. Put

$$
\begin{gathered}
\Pi\left(g_{0}\right)=H^{g_{0}}=H_{0}, \\
\Pi\left(g_{k}\right)=H^{g_{k}}=H_{p_{1}} \oplus H_{p_{2}} \oplus \ldots \oplus H_{p_{k}}, \\
\Pi\left(g_{k-1}\right)=H^{g_{k-1}}=H_{p_{2}} \oplus \ldots \oplus H_{p_{k-1}} \oplus H_{p_{k}}, \\
\Pi\left(g_{k-2}\right)=H^{g_{k-2}}=H_{p_{2}} \oplus H_{p_{3}} \oplus \ldots \oplus H_{p_{k-1}},
\end{gathered}
$$

In these equalities the summands are omitted from the left and the right in turns. Analogously, we define subspaces $\Pi\left(g_{i}\right)$ for $i=k+1, \ldots, k+l$ and $i=k+l+1, \ldots, k+$ $l+m$. Define the operators $\Gamma_{g_{0}, g_{i}}: H^{g_{i}} \rightarrow H^{g_{0}}$, where $i \in\{k, k+l, k+l+m\}$, by 
the block-diagonal matrices

$$
\begin{gathered}
\Gamma_{g_{0}, g_{k}}=\left[\sqrt{\alpha_{1}} \Gamma_{p_{1}}\left|\sqrt{\alpha_{2}} \Gamma_{p_{2}}\right| \ldots \mid \sqrt{\alpha_{k}} \Gamma_{p_{k}}\right], \\
\Gamma_{g_{0}, g_{k+l}}=\left[\sqrt{\beta_{1}} \Gamma_{q_{1}}\left|\sqrt{\beta_{2}} \Gamma_{q_{2}}\right| \ldots \mid \sqrt{\beta_{l}} \Gamma_{q_{l}}\right], \\
\Gamma_{g_{0}, g_{k+l+m}}=\left[\sqrt{\delta_{1}} \Gamma_{s_{1}}\left|\sqrt{\delta_{2}} \Gamma_{s_{2}}\right| \ldots \mid \sqrt{\delta_{m}} \Gamma_{s_{m}}\right] .
\end{gathered}
$$

Now we define the representation $\Pi$ on the edges $\gamma_{g_{0}, g_{k}}, \gamma_{g_{0}, g_{k+l}}, \gamma_{g_{0}, g_{k+l+m}}$ by the rule

$$
\begin{gathered}
\Pi\left(\gamma_{g_{0}, g_{k}}\right)=\left\{\Gamma_{g_{0}, g_{k}}, \Gamma_{g_{k}, g_{0}}\right\}, \\
\Pi\left(\gamma_{g_{0}, g_{k+l}}\right)=\left\{\Gamma_{g_{0}, g_{k+l}}, \Gamma_{g_{k+l}, g_{0}}\right\}, \\
\Pi\left(\gamma_{g_{0}, g_{k+l+m}}\right)=\left\{\Gamma_{g_{0}, g_{k+l+m}}, \Gamma_{g_{k+l+m}, g_{0}}\right\} .
\end{gathered}
$$

It is easy to see that

$$
\Gamma_{g_{0}, g_{k}} \Gamma_{g_{k}, g_{0}}+\Gamma_{g_{0}, g_{k+l}} \Gamma_{g_{k+l}, g_{0}}+\Gamma_{g_{0}, g_{k+l+m}} \Gamma_{g_{k+l+m}, g_{0}}=\gamma I_{H^{g_{0}}} .
$$

Let $\mathcal{O}_{H, 0}$ denote the operators from the zero space to $H$, and $\mathcal{O}_{0, H}$ denote the zero operator from $H$ into the zero subspace. For the operators $\Gamma_{g_{j}, g_{i}}: H^{g_{i}} \rightarrow H^{g_{j}}$ with $i, j \neq 0$, put

$$
\begin{aligned}
\Gamma_{g_{k-1}, g_{k}} & =\mathcal{O}_{0, H_{p_{1}}} \oplus \sqrt{\alpha_{1}-\alpha_{2}} I_{H_{p_{2}}} \oplus \sqrt{\alpha_{1}-\alpha_{3}} I_{H_{p_{3}}} \oplus \ldots \oplus \sqrt{\alpha_{1}-\alpha_{k}} I_{H_{p_{k}}}, \\
\Gamma_{g_{k-1}, g_{k-2}} & =\sqrt{\alpha_{2}-\alpha_{k}} I_{H_{p_{2}}} \oplus \sqrt{\alpha_{3}-\alpha_{k}} I_{H_{p_{3}}} \oplus \ldots \oplus \sqrt{\alpha_{k-1}-\alpha_{k}} I_{H_{p_{k-1}}} \oplus \mathcal{O}_{H_{p_{k}}, 0}, \\
\Gamma_{g_{k-3}, g_{k-2}} & =\mathcal{O}_{0, H_{p_{2}}} \oplus \sqrt{\alpha_{2}-\alpha_{3}} I_{H_{p_{3}}} \oplus \sqrt{\alpha_{2}-\alpha_{4}} I_{H_{p_{4}}} \oplus \ldots \oplus \sqrt{\alpha_{2}-\alpha_{k-1}} I_{H_{p_{k-1}}},
\end{aligned}
$$

The corresponding operators for the rest of the edges of $G$ can be constructed analogously. One can check that the operators $\Gamma_{g_{i}, g_{j}}$, where $\Gamma_{g_{i}, g_{j}}=\Gamma_{g_{i}, g_{j}}^{*}$, define a locally-scalar representation of the graph $G$ with the following character $f$ :

$$
\begin{aligned}
& f\left(g_{k}\right)=\alpha_{1}, \quad f\left(g_{k+l}\right)=\beta_{1}, \quad f\left(g_{k+l+m}\right)=\delta_{1}, \\
& f\left(g_{k-1}\right)=\alpha_{1}-\alpha_{k}, \quad f\left(g_{k+l-1}\right)=\beta_{1}-\beta_{l}, \quad f\left(g_{k+l+m-1}\right)=\delta_{1}-\delta_{m}, \\
& f\left(g_{k-2}\right)=\alpha_{2}-\alpha_{k}, \quad f\left(g_{k+l-2}\right)=\beta_{2}-\beta_{l}, \quad f\left(g_{k+l+m-2}\right)=\delta_{2}-\delta_{m}, \\
& f\left(g_{k-3}\right)=\alpha_{2}-\alpha_{k-1}, \quad f\left(g_{k+l-3}\right)=\beta_{2}-\beta_{l-1}, \quad f\left(g_{k+l+m-3}\right)=\delta_{2}-\delta_{m-1}, \\
& f\left(g_{k-4}\right)=\alpha_{3}-\alpha_{k-1}, \quad f\left(g_{k+l-4}\right)=\beta_{3}-\beta_{l-1}, \quad f\left(g_{k+l+m-4}\right)=\delta_{3}-\delta_{m-1}, \\
& f\left(g_{0}\right)=\gamma
\end{aligned}
$$


And vice versa, if a locally-scalar representation of the graph $G$ with the character $f\left(g_{i}\right)=x_{i} \in \mathbb{R}^{*}$ corresponds to a non-degenerate representation of $\mathcal{P}_{\alpha, \beta, \delta, \gamma}$, then one can check that

$$
\begin{gathered}
\alpha_{1}=x_{k}, \\
\alpha_{k}=x_{k}-x_{k-1}, \alpha_{2}=x_{k}-x_{k-1}+x_{k-2}, \\
\alpha_{k-1}=x_{k}-x_{k-1}+x_{k-2}-x_{k-3}, \\
\alpha_{3}=x_{k}-x_{k-1}+x_{k-2}-x_{k-3}+x_{k-4},
\end{gathered}
$$

Here $x_{j}=0$ if $j \leq 0$. Analogously one can find $\beta_{j}$ and $\delta_{t}$. We will denote $\Pi$ by $\Phi(\pi)$.

Let $\pi$ and $\tilde{\pi}$ be non-degenerate representations of the algebra $\mathcal{P}_{\alpha, \beta, \delta, \gamma}$ and $C_{0}$ an intertwining operator for these representations; this is a morphism from $\pi$ to $\widetilde{\pi}$ in the category $\operatorname{Rep} G), C_{0}: H_{0} \rightarrow \widetilde{H}_{0}, C_{0} \pi=\widetilde{\pi} C_{0}$. Put

$$
\begin{gathered}
C_{p_{i}}=\widetilde{\Gamma}_{p_{i}}^{*} C_{0} \Gamma_{p_{i}}, C_{p_{i}}: H_{p_{i}} \rightarrow \widetilde{H}_{p_{i}}, 1 \leq i \leq k, \\
C_{q_{j}}=\widetilde{\Gamma}_{q_{j}}^{*} C_{0} \Gamma_{q_{j}}, C_{q_{j}}: H_{q_{j}} \rightarrow \widetilde{H}_{q_{j}}, k+1 \leq j \leq k+l, \\
C_{s_{t}}=\widetilde{\Gamma}_{s_{t}}^{*} C_{0} \Gamma_{s_{t}}, C_{s_{t}}: H_{s_{t}} \rightarrow \widetilde{H}_{s_{t}} . k+l+1 \leq t \leq k+l+m,
\end{gathered}
$$

Put

$$
\begin{gathered}
C^{\left(g_{0}\right)}=C_{0}: H^{\left(g_{0}\right)} \rightarrow \widetilde{H}^{\left(g_{0}\right)}, \\
C^{\left(g_{k}\right)}=C_{p_{1}} \oplus C_{p_{2}} \oplus \ldots \oplus C_{p_{k}}: H^{\left(g_{k}\right)} \rightarrow \widetilde{H}^{\left(g_{k}\right)}, \\
C^{\left(g_{k-1}\right)}=C_{p_{2}} \oplus \ldots \oplus C_{p_{k-1}} \oplus C_{p_{k}}: H^{\left(g_{k-1}\right)} \rightarrow \widetilde{H}^{\left(g_{k-1}\right)}, \\
C^{\left(g_{k-2}\right)}=C_{p_{2}} \oplus C_{p_{3}} \oplus \ldots \oplus C_{p_{k-1}}: H^{\left(g_{k-2}\right)} \rightarrow \widetilde{H}^{\left(g_{k-2}\right)},
\end{gathered}
$$

Analogously one can construct the operators $C^{\left(g_{i}\right)}$ for $i \in\{k+l, \ldots, k+l+m\}$. It is routine to check that the operators $\left\{C^{\left(g_{i}\right)}\right\}_{0 \leq i \leq k+l+m}$ intertwine the representations $\Pi=\Phi(\pi)$ and $\widetilde{\Pi}=\Phi(\widetilde{\pi})$. Put $\Phi\left(C_{0}\right)=\left\{C_{0 \leq i \leq k+l+m}^{\left(g_{i}\right)}\right\}$. Thus we have defined a functor $\Phi: \overline{\operatorname{Rep}} \mathcal{P}_{\alpha, \beta, \delta, \gamma} \rightarrow \operatorname{Rep} G$, see [5]. Moreover, the functor $\Phi$ is univalent and full. Let $\widehat{\operatorname{Rep}}(G, d, f)$ be the full subcategory of irreducible representations of $\operatorname{Rep}(G, d, f) . \quad \Pi \in \operatorname{Ob} \widehat{\operatorname{Rep}}(G, d, f), f\left(g_{i}\right)=x_{i} \in \mathbb{R}^{+}, d\left(g_{i}\right)=d_{i} \in \mathbb{N}_{0}$, where $f$ is the character of $\Pi, d$ its dimension. It easy to verify that the representation $\Pi$ is isomorphic (unitary equivalent) to an irreducible representation from the image of the functor $\Phi$ if and only if

$$
\begin{gathered}
\text { 1. } 0<x_{1}<x_{2}<\ldots<x_{k} ; 0<x_{k+1}<x_{k+2}<\ldots<x_{k+l} ; \\
0<x_{k+l+1}<x_{k+l+2}<\ldots<x_{k+l+m} \\
\text { 2. } 0<d_{1}<d_{2}<\ldots<d_{k}<d_{0} ; 0<d_{k+1}<d_{k+2}<\ldots< \\
d_{k+l}<d_{0} ; 0<d_{k+l+1}<d_{k+l+2}<\ldots<d_{k+l+m}<d_{0} .
\end{gathered}
$$


(All matrices of the representation of the graph $G$, except for $\Gamma_{g_{0}, g_{k}}, \Gamma_{g_{k}, g_{0}}, \Gamma_{g_{0}, g_{k+l}}$, $\Gamma_{g_{k+l}, g_{0}}, \Gamma_{g_{0}, g_{k+l+m}}, \Gamma_{g_{k+l+m}, g_{0}}$, can be brought to the "canonical" form (2.1) by admissible transformations. Then the rest of the matrices will naturally be partitioned into blocks, which gives the matrices $\Gamma_{p_{i}}, \Gamma_{q_{i}}, \Gamma_{s_{i}}$, and thus the projections $P_{i}, Q_{i}, R_{i}$ ). An irreducible representation $\Pi$ of the graph $G$ satisfying conditions (2.2)-(2.5) will be called non-degenerate. Let

$$
\begin{gathered}
\operatorname{dim} H_{p_{i}}=n_{i}, 1 \leq i \leq k ; \\
\operatorname{dim} H_{q_{j}}=n_{k+j}, 1 \leq j \leq l ; \\
\operatorname{dim} H_{s_{t}}=n_{k+l+t}, 1 \leq t \leq m ; \\
\operatorname{dim} H_{0}=n_{0}
\end{gathered}
$$

The vector $n=\left(n_{0}, n_{1}, \ldots, n_{k+l+m}\right)$ is called a generalized dimension of the representation $\pi$ of the algebra $\mathcal{P}_{\alpha, \beta, \delta, \gamma}$. Let $\Pi=\Phi(\pi)$ for a non-degenerate representation of the algebra $\mathcal{P}_{\alpha, \beta, \delta, \gamma}, d=\left(d_{0}, d_{1}, \ldots, d_{k+l+m}\right)$ be the dimension of $\Pi$. It is easy to see that

$$
\begin{gathered}
n_{1}+n_{2}+\ldots+n_{k}=d_{k}, \\
n_{2}+\ldots+n_{k-1}+n_{k}=d_{k-1}, \\
n_{2}+\ldots+n_{k-1}=d_{k-2}, \\
n_{3}+\ldots+n_{k-2}+n_{k-1}=d_{k-3},
\end{gathered}
$$

Thus

$$
\begin{gathered}
n_{1}=d_{k}-d_{k-1}, \\
n_{k}=d_{k-1}-d_{k-2}, \\
n_{2}=d_{k-2}-d_{k-3},
\end{gathered}
$$$$
\ldots
$$

Analogously one can find $n_{k+1}, \ldots, n_{k+l}$ from $d_{k+1}, \ldots, d_{k+l}$ and $n_{k+l+1}, \ldots, n_{k+l+m}$ from $d_{k+l+1}, \ldots, d_{k+l+m}$

Denote by $\overline{\operatorname{Rep}} G$ the full subcategory in $\operatorname{Rep} G$ of non-degenerate locally-scalar representations of the graph $G$. As a corollary of the previous arguments we obtain the following theorem.

Theorem 1 Let $\mathcal{P}_{\alpha, \beta, \delta, \gamma}$ be associated with a graph $G$. The functor $\Phi$ is an equivalence of the categories $\overline{\operatorname{Rep}} \mathcal{P}_{\alpha, \beta, \delta, \gamma}$ of non-degenerate ${ }^{*}$-representations of the algebra $\mathcal{P}_{\alpha, \beta, \delta, \gamma}$ and the category $\overline{\operatorname{Rep}} G$ of non-degenerate locally-scalar representations of the graph $G$.

Let us define the Coxeter functors for the $*$-algebras $\mathcal{P}_{\alpha, \beta, \delta, \gamma}$, by putting $\stackrel{\circ}{\Psi}=$ $\Phi^{-1} \stackrel{\circ}{F} \Phi$ and $\stackrel{\bullet}{\Psi}=\Phi^{-1} \dot{\circ} \Phi$. Now we can use the results of [6] to give a description of representation of the ${ }^{*}$-algebra $\mathcal{P}_{\alpha, \beta, \delta, \gamma}$. 
Obviously, all irreducible representations of the *-algebra associated with the Dynkin diagram $A_{n}$ are one-dimensional, with diagram $D_{n}$ one- or two-dimensional and only the algebras associated with the diagrams $D_{4}, E_{6}, E_{7}, E_{8}$ have non-degenerate irreducible representations.

In the next section we will do the following: we know how to construct all irreducible locally-scalar representations of Dynkin graphs with the aid of Coxeter reflection functors starting from the simplest ones. In particular, we can find their dimensions and characters [6]. Next we single out non-generate representations and apply the equivalence functor $\Phi$, see Theorem 1 .

\section{Algebras associated with Dynkin graphs.}

Let $G$ be the Dynkin graph $E_{6}$,

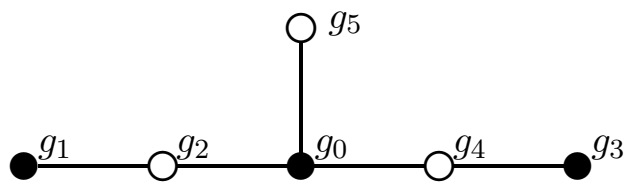

The vertices $g_{0}, g_{1}, g_{3}$ will be called odd and marked with $\bullet$ on the graph, the vertices $g_{2}, g_{4}, g_{5}$ are even and indicated with $\circ$. The parameters of the corresponding algebra $\mathcal{P}_{\alpha, \beta, \delta, \gamma}$ are enumerated according to the following picture:

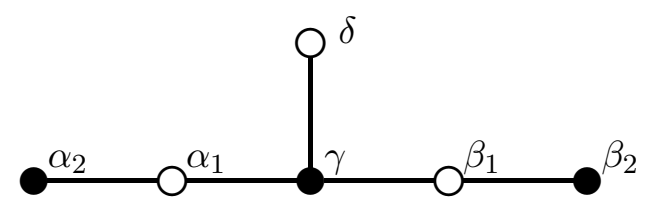

Using the proof of theorem 3.9 (see [6]) and direct calculations we see that all nondegenerate representations of the graph $E_{6}$ can be obtained by the Coxeter reflection

functors $\stackrel{\circ}{F}$ and $\dot{F}$ in 4 and 5 steps from the simplest one $\Pi_{g_{0}}$ that corresponds to the simple root $\bar{g}_{0}$ of the graph $E_{6}$. The dimension of the representation $\Pi_{g_{0}}$ is denoted by $d_{g_{0}}(g)$. Then $d_{g_{0}}\left(g_{i}\right)=0$ if $g_{i} \neq g_{0}, d_{g_{0}}\left(g_{0}\right)=1$. Let the character of $\Pi_{g_{0}}$ is denoted by $f_{g_{0}}$, then $f_{g_{0}}\left(g_{0}\right)=0, f_{g_{0}}\left(g_{i}\right)=t_{i}>0$ if $g_{i} \neq g_{0}$. All irreducible locally-scalar representations of Dynkin diagrams are obtained from the simple representations with characters of such type by using the Coxeter reflection functors (see [6]). In the subsequent calculations we will write the character value below or to the right and the dimension over or to the left of the vertex.

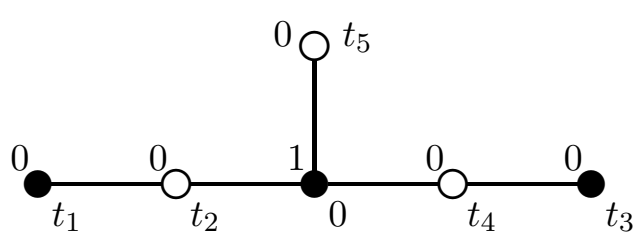


Thus $\Pi=\stackrel{\circ}{F}_{4}\left(\Pi_{g_{0}}\right)$ is a non-degenerate representation of the graph $G$ with dimension $d=\stackrel{\circ}{c}_{4}\left(d_{g_{0}}\right)$. Calculations values of the dimensions are summarized in the following table:

\begin{tabular}{|c|c|c|c|c|c|c|}
\hline$g_{i}$ & $g_{0}$ & $g_{1}$ & $g_{2}$ & $g_{3}$ & $g_{4}$ & $g_{5}$ \\
\hline$d$ & 3 & 1 & 2 & 1 & 2 & 1 \\
\hline
\end{tabular}

Then $\Pi_{g_{0}}=\dot{\circ}_{4}(\Pi), d_{g_{0}}=\dot{\bullet}_{4}(d)$ i $f_{g_{0}}(g)=\stackrel{\circ}{c_{d}}(f)$.

Thus

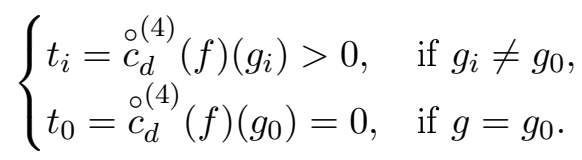

Let $\pi$ be a non-degenerate representation of the algebra $\mathcal{P}_{\alpha, \beta, \delta, \gamma}$. Then $\Phi(\pi)$ is non-degenerate representation from $\overline{\operatorname{Rep}} G$ with the character $f$ given by the following table (see equalities (2) ):

\begin{tabular}{|c|c|c|c|c|c|c|}
\hline$g_{i}$ & $g_{0}$ & $g_{1}$ & $g_{2}$ & $g_{3}$ & $g_{4}$ & $g_{5}$ \\
\hline$f$ & $\gamma$ & $\alpha_{1}-\alpha_{2}$ & $\alpha_{1}$ & $\beta_{1}-\beta_{2}$ & $\beta_{1}$ & $\delta$ \\
\hline
\end{tabular}

Conditions (3.1) imposed on $f\left(g_{i}\right)$ are necessary and sufficient for a representation of the algebra $\mathcal{P}_{\alpha, \beta, \delta, \gamma}$ in the dimension $\Phi(d)$ to exist. Computing the character we obtain $\stackrel{\circ(4)}{c_{d}}(f)$, and from (3.1) we get the following inequalities:

$$
\gamma>\alpha_{1}, \gamma>\beta_{1}, \alpha_{2}+\beta_{1}>\gamma, \alpha_{1}+\beta_{2}>\gamma, \gamma>\alpha_{2}+\beta_{2}
$$

and the identity $3 \gamma=\delta+\alpha_{1}+\alpha_{2}+\beta_{1}+\beta_{2}$. Knowing the dimension $d$ we find the corresponding generalized dimension $\Phi^{-1}(d)=\left(n_{g_{0}} ; n_{g_{1}}, \ldots, n_{g_{5}}\right)$ of the representation $\pi$, see formulae (2.6), where $n_{g_{i}}=n_{i}$.

\begin{tabular}{|c|c|c|c|c|c|c|}
\hline$g_{i}$ & $g_{0}$ & $g_{1}$ & $g_{2}$ & $g_{3}$ & $g_{4}$ & $g_{5}$ \\
\hline$\Phi^{-1}(d)$ & 3 & 1 & 1 & 1 & 1 & 1 \\
\hline
\end{tabular}

In a similar way, one can perform calculations for the representation $\stackrel{\circ}{F}_{5}\left(\Pi_{g_{0}}\right)$, the only remaining non-degenerate irreducible representation of the Dynkin graph $E_{6}$. Thus we get the following theorem.

Theorem 2 The algebra $\mathcal{P}_{\left(\alpha_{1}, \alpha_{2}\right),\left(\beta_{1}, \beta_{2}\right), \delta, \gamma}$ associated with the Dynkin graph $E_{6}$ has an irreducible non-degenerate representation only in the following cases.

1. The following conditions are satisfied:

$$
\gamma>\alpha_{1}, \gamma>\beta_{1}, \alpha_{2}+\beta_{1}>\gamma, \alpha_{1}+\beta_{2}>\gamma, \gamma>\alpha_{2}+\beta_{2},
$$

and $3 \gamma=\delta+\alpha_{1}+\alpha_{2}+\beta_{1}+\beta_{2}$; in this case there is a unique representation in the generalized dimension $(3 ; 1,1,1,1,1)$

2. The following conditions are satisfied:

$$
\delta+\beta_{1}>\gamma, \gamma>\delta+\alpha_{2}, \gamma>\delta+\beta_{2}, \delta+\alpha_{1}>\gamma, \delta+\alpha_{2}+\beta_{2}>\gamma,
$$

and $3 \gamma=2 \delta+\alpha_{1}+\alpha_{2}+\beta_{1}+\beta_{2}$; in this case there is a unique representation in the generalized dimension $(3 ; 1,1,1,1,2)$ 
We have shown in the example of $E_{6}$ how to perform calculations and derive conditions of existence for an irreducible non-degenerate representation of the corresponding *-algebra. Now without additional explanations we present complete answers for the rest of the Dynkin graphs. We label vertices $g_{i}$ of the graph $G$ as in the following pictures and write the corresponding parameters of the algebra in parentheses:
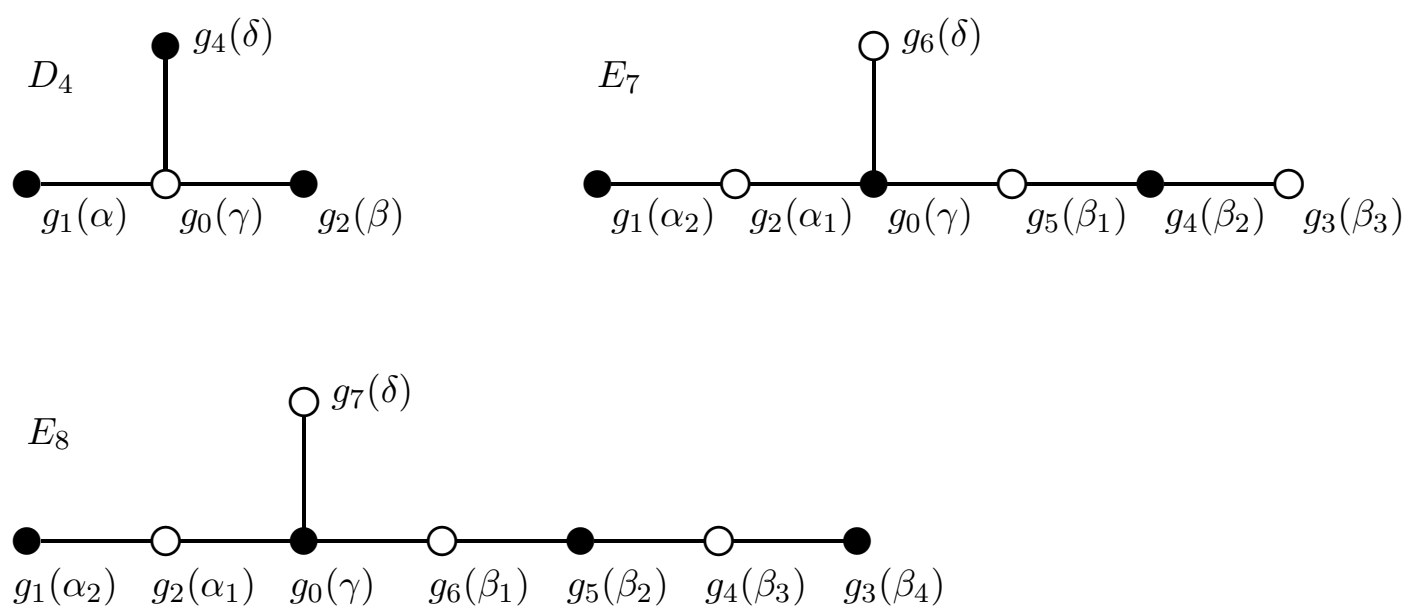

This correspondence is important and henceforth we will designate the coordinates of the generalized dimension vectors by the vertices of the graph $G, \Phi^{-1}(d)=$ $\left(n_{g_{0}} ; n_{g_{1}}, n_{g_{2}}, \ldots, n_{g_{k+l+m}}\right)$. The following theorem holds.

Theorem 3 The algebra $\mathcal{P}_{\alpha, \beta, \delta, \gamma}$ associated with $D_{4}$ has an irreducible non-degenerate ${ }^{*}$-representation only in the dimension $(2 ; 1,1,1)$. This representation is unique and exists if and only if the following conditions are satisfied:

$$
\begin{gathered}
\delta+\beta>\alpha, \alpha+\beta>\delta, \delta+\alpha>\beta, \\
2 \gamma=\alpha+\beta+\delta .
\end{gathered}
$$

All irreducible non-degenerate locally-scalar representations of the graph $E_{7}$ are obtained from the simplest representation $\Pi_{g_{0}}$ : in 6 steps we obtain a non-degenerate representation of dimension ${\stackrel{\circ}{c_{6}}}_{6}\left(d_{g_{0}}\right)=(4 ; 1,2 ; 1,2,3 ; 2)$, and in 7 steps - of dimension $\stackrel{\circ}{c}_{7}\left(d_{g_{0}}\right)=(4 ; 1,3 ; 1,2,3 ; 2)$, in 8 steps of dimension $\stackrel{\circ}{c}_{8}\left(d_{g_{0}}\right)=(4 ; 2,3 ; 1,2,3 ; 2)$ (these are dimensions $d$ of the graph representations. Using them the corresponding dimensions of the algebra representations can be computed as $\Phi^{-1}(d)$ ). The following theorem holds.

Theorem 4 The algebra $\mathcal{P}_{\alpha, \beta, \delta, \gamma}$ associated with the graph $E_{7}$ has an irreducible non-degenerate *-representation only in the following cases.

1. In dimension $(4 ; 1,1 ; 1,1,1 ; 2)$, provided that

$$
\begin{aligned}
& \qquad \begin{aligned}
\gamma & >\delta+\beta_{3}, \delta+\alpha_{1}+\beta_{1}>2 \gamma, \delta+\beta_{2}>\gamma, \\
2 \gamma & >\delta+\alpha_{2}+\beta_{1}, \gamma>\beta_{1}, 2 \gamma>\delta+\alpha_{1}+\beta_{2},
\end{aligned} \\
& \text { and } 4 \gamma=\alpha_{1}+\alpha_{2}+\beta_{1}+\beta_{2}+\beta_{3}+2 \delta .
\end{aligned}
$$


2. In dimension $(4 ; 1,2 ; 1,1,1 ; 2)$, provided that

$$
\begin{gathered}
\alpha_{1}+\beta_{2}>\gamma, 2 \gamma>\alpha_{1}+\beta_{1}+\delta, \gamma>\alpha_{1}+\beta_{3}, 2 \gamma>\delta+\alpha_{1}+\beta_{2}+\beta_{3}, \\
\delta+\alpha_{1}>\gamma, \delta+\alpha_{1}+\beta_{1}+\beta_{3}>2 \gamma, \\
4 \gamma=2 \alpha_{1}+\alpha_{2}+\beta_{1}+\beta_{2}+\beta_{3}+2 \delta .
\end{gathered}
$$

3. In dimension $(4 ; 2,1 ; 1,1,1 ; 2)$, provided that

$$
\begin{gathered}
\gamma>\alpha_{2}+\beta_{2}, \delta+\alpha_{2}+\beta_{3}>\gamma, \alpha_{2}+\beta_{1}>\gamma, \delta+\alpha_{2}+\beta_{1}+\beta_{2}>2 \gamma, \\
\gamma>\delta+\alpha_{2}, 2 \gamma>\delta+\alpha_{2}+\beta_{1}+\beta_{3}, \\
4 \gamma=\alpha_{1}+2 \alpha_{2}+\beta_{1}+\beta_{2}+\beta_{3}+2 \delta .
\end{gathered}
$$

In each of the above cases, the representation is unique up to unitary equivalence.

Non-degenerate irreducible representations of the graph $E_{8}$ are obtained from the simplest ones $\Pi_{g_{0}}$ and $\Pi_{g_{6}}$. In $k$ steps, where $k=9,10, \ldots, 14$, starting from $\Pi_{g_{0}}$ we obtain a non-degenerate representation of dimension $\stackrel{\circ}{c}_{k}\left(d_{g_{0}}\right)$. Analogously, in $k$ steps, where $k=11,12,13,14$, using $\Pi_{g_{6}}$ we obtain a non-degenerate representation of dimension $\stackrel{\circ}{c}_{k}\left(d_{g_{6}}\right)$, where $d_{g_{6}}\left(g_{6}\right)=1$ and $d_{g_{6}}\left(g_{i}\right)=0$, if $g_{i} \neq g_{6}$.

Theorem 5 The algebra $\mathcal{P}_{\alpha, \beta, \delta, \gamma}$ associated with graph $E_{8}$ has an irreducible nondegenerate ${ }^{*}$-representation only in the following cases.

1. In dimension $(5 ; 2,1 ; 1,1,1,1 ; 2)$, provided that

$$
\begin{gathered}
\gamma>\alpha_{2}+\beta_{3}, \delta+\alpha_{2}+\beta_{1}+\beta_{4}>2 \gamma, \alpha_{2}+\beta_{2}>\gamma, \\
\delta+\alpha_{2}+\beta_{2}+\beta_{3}>2 \gamma, 2 \gamma>\delta+\alpha_{2}+\beta_{1}, \gamma>\beta_{1}, \\
2 \gamma>\delta+\alpha_{2}+\beta_{2}+\beta_{4}, \\
\gamma=\frac{1}{5}\left(2 \delta+\alpha_{1}+2 \alpha_{2}+\beta_{1}+\beta_{2}+\beta_{3}+\beta_{4}\right) .
\end{gathered}
$$

2. In dimension $(5 ; 2,2 ; 1,1,1,1 ; 3)$, provided that

$$
\begin{gathered}
\delta+\alpha_{2}+\beta_{3}>\gamma, 5 \gamma+\beta_{1}+\beta_{3}+\beta_{4}>2 \delta+3 \alpha_{1}+3 \alpha_{2}+4 \beta_{2}, \\
\gamma>\delta+\alpha_{2}+\beta_{4}, 4 \delta+\alpha_{1}+\alpha_{2}+3 \beta_{1}+3 \beta_{2}>5 \gamma+2 \beta_{3}+2 \beta_{4}, \\
2 \delta+3 \alpha_{1}+3 \alpha_{2}+4 \beta_{1}>5 \gamma+\beta_{2}+\beta_{3}+\beta_{4}, \delta+\alpha_{1}>\gamma, \\
2 \delta+3 \alpha_{1}+3 \alpha_{2}+4 \beta_{2}+4 \beta_{4}>5 \gamma+\beta_{1}+\beta_{3}, \\
\gamma=\frac{1}{5}\left(3 \delta+2 \alpha_{1}+2 \alpha_{2}+\beta_{1}+\beta_{2}+\beta_{3}+\beta_{4}\right) .
\end{gathered}
$$

3. In dimension $(6 ; 2,2 ; 1,1,1,1 ; 3)$, provided that

$$
\begin{gathered}
2 \gamma>\delta+\alpha_{1}+\beta_{2}, \delta+\alpha_{1}+\alpha_{2}+\beta_{3}>2 \gamma, \delta+\alpha_{1}+\beta_{1}>2 \gamma, \\
3 \gamma>2 \delta+\alpha_{1}+\alpha_{2}+\beta_{3}+\beta_{4}, 2 \gamma>\delta+\alpha_{1}+\alpha_{2}+\beta_{4}, \gamma>\delta+\alpha_{2}, \\
2 \delta+\alpha_{1}+\alpha_{2}+\beta_{2}+\beta_{4}>3 \gamma, \\
\gamma=\frac{1}{6}\left(3 \delta+2 \alpha_{1}+2 \alpha_{2}+\beta_{1}+\beta_{2}+\beta_{3}+\beta_{4}\right) .
\end{gathered}
$$


4. In dimension $(6 ; 2,2 ; 1,1,1,2 ; 3)$, provided that

$$
\begin{gathered}
\delta+\alpha_{1}+\beta_{1}+\beta_{4}>2 \gamma, 2 \gamma>\delta+\alpha_{2}+\beta_{1}+\beta_{3}, 2 \gamma>\delta+\alpha_{1}+\beta_{1}, \\
3 \gamma>2 \delta+\alpha_{1}+\alpha_{2}+\beta_{1}+\beta_{4}, \delta+\alpha_{2}+\beta_{1}+\beta_{2}>2 \gamma, \alpha_{2}+\beta_{1}>\gamma, \\
2 \delta+\alpha_{1}+\alpha_{2}+\beta_{1}+\beta_{3}>3 \gamma, \\
\gamma=\frac{1}{6}\left(3 \delta+2 \alpha_{1}+2 \alpha_{2}+2 \beta_{1}+\beta_{2}+\beta_{3}+\beta_{4}\right) .
\end{gathered}
$$

5. In dimension $(6 ; 2,2 ; 2,1,1,1 ; 3)$, provided that

$$
\begin{gathered}
2 \gamma>\delta+\alpha_{2}+\beta_{1}+\beta_{4}, \delta+\alpha_{1}+\beta_{2}+\beta_{4}>2 \gamma, \delta+\alpha_{2}+\beta_{4}>\gamma, \\
2 \delta+\alpha_{1}+\alpha_{2}+\beta_{1}+\beta_{4}>3 \gamma, 2 \gamma>\delta+\alpha_{1}+\beta_{3}+\beta_{4}, \gamma>\alpha_{1}+\beta_{4}, \\
3 \gamma>2 \delta+\alpha_{1}+\alpha_{2}+\beta_{2}+\beta_{4}, \\
\gamma=\frac{1}{6}\left(3 \delta+2 \alpha_{1}+2 \alpha_{2}+\beta_{1}+\beta_{2}+\beta_{3}+2 \beta_{4}\right) .
\end{gathered}
$$

6. In dimension $(6 ; 2,2 ; 1,1,2,1 ; 3)$, provided that

$$
\begin{gathered}
\delta+\alpha_{1}+\alpha_{2}+\beta_{2}>2 \gamma, 2 \gamma>\delta+\alpha_{1}+\beta_{2}+\beta_{4}, \gamma>\alpha_{2}+\beta_{2}, \\
3 \gamma>\delta+\alpha_{1}+\alpha_{2}+\beta_{1}+\beta_{2}, \delta+\alpha_{1}+\beta_{2}+\beta_{3}>2 \gamma, \delta+\beta_{2}>\gamma, \\
3 \gamma>2 \delta+\alpha_{1}+\alpha_{2}+\beta_{2}+\beta_{3}, \\
\gamma=\frac{1}{6}\left(3 \delta+2 \alpha_{1}+2 \alpha_{2}+\beta_{1}+2 \beta_{2}+\beta_{3}+\beta_{4}\right) .
\end{gathered}
$$

7. In dimension $(6 ; 2,2 ; 1,2,1,1 ; 3)$, provided that

$$
\begin{gathered}
2 \gamma>\delta+\alpha_{1}+\alpha_{2}+\beta_{3}, \delta+\alpha_{2}+\beta_{1}+\beta_{3}>2 \gamma, \alpha_{1}+\beta_{3}>\gamma, \\
\delta+\alpha_{1}+\alpha_{2}+\beta_{3}+\beta_{4}>2 \gamma, 2 \gamma>\delta+\alpha_{2}+\beta_{2}+\beta_{3}, \gamma>\delta+\beta_{3}, \\
2 \delta+\alpha_{1}+\alpha_{2}+\beta_{2}+\beta_{3}>3 \gamma, \\
\gamma=\frac{1}{6}\left(3 \delta+2 \alpha_{1}+2 \alpha_{2}+\beta_{1}+\beta_{2}+2 \beta_{3}+\beta_{4}\right) .
\end{gathered}
$$

8. In dimension $(5 ; 1,2 ; 1,1,1,1 ; 2)$, provided that

$$
\begin{gathered}
5 \gamma+\alpha_{2}+\beta_{1}+\beta_{3}+\beta_{4}>3 \delta+3 \alpha_{1}+4 \beta_{2}, \alpha_{1}+\beta_{3}>\gamma, \gamma>\beta_{1}, \\
5 \gamma+2 \beta_{1}>\delta+\alpha_{1}+3 \alpha_{2}+3 \beta_{2}+3 \beta_{3}+3 \beta_{4}, \\
5 \gamma+\alpha_{2}+\beta_{1}+\beta_{2}>3 \delta+3 \alpha_{1}+4 \beta_{3}+4 \beta_{4}, \gamma>\alpha_{1}+\beta_{4}, \\
5 \gamma+2 \beta_{2}+2 \beta_{4}>\delta+\alpha_{1}+3 \alpha_{2}+3 \beta_{1}+3 \beta_{3}, \\
\gamma=\frac{1}{5}\left(2 \delta+2 \alpha_{1}+\alpha_{2}+\beta_{1}+\beta_{2}+\beta_{3}+\beta_{4}\right) .
\end{gathered}
$$

9. In dimension $(5 ; 1,2 ; 1,1,1,1 ; 3)$, provided that

$$
\begin{gathered}
5 \gamma+2 \beta_{1}+2 \beta_{4}>4 \delta+\alpha_{1}+3 \alpha_{2}+3 \beta_{2}+3 \beta_{3}, \gamma>\delta+\beta_{3}, \\
\delta+\alpha_{1}>\gamma, 5 \gamma+\alpha_{2}+\beta_{2}+\beta_{3}+\beta_{4}>2 \delta+3 \alpha_{1}+4 \beta_{1}, \\
5 \gamma+2 \beta_{2}+2 \beta_{3}>4 \delta+\alpha_{1}+3 \alpha_{2}+3 \beta_{1}+3 \beta_{4}, \delta+\beta_{2}>\gamma \\
5 \gamma+\alpha_{2}+\beta_{1}+\beta_{3}>2 \delta+3 \alpha_{1}+4 \beta_{2}+4 \beta_{4}, \\
\gamma=\frac{1}{5}\left(3 \delta+2 \alpha_{1}+\alpha_{2}+\beta_{1}+\beta_{2}+\beta_{3}+\beta_{4}\right) .
\end{gathered}
$$


10. In dimension $(5 ; 2,1 ; 1,1,1,1 ; 3)$, provided that

$$
\begin{gathered}
5 \gamma+\alpha_{1}+\beta_{2}+\beta_{3}>2 \delta+3 \alpha_{2}+4 \beta_{1}+4 \beta_{4}, \delta+\beta_{2}>\gamma \\
\gamma>\delta+\alpha_{2}, \delta+\alpha_{2}+\beta_{4}>\gamma, 5 \gamma+\alpha_{1}+\beta_{1}+\beta_{4}>2 \delta+3 \alpha_{2}+4 \beta_{2}+4 \beta_{3} \\
\gamma>\delta+\beta_{3}, 5 \gamma+2 \beta_{1}+2 \beta_{3}>4 \delta+3 \alpha_{1}+\alpha_{2}+3 \beta_{2}+3 \beta_{4} \\
\gamma=\frac{1}{5}\left(3 \delta+\alpha_{1}+2 \alpha_{2}+\beta_{1}+\beta_{2}+\beta_{3}+\beta_{4}\right) .
\end{gathered}
$$

11. In dimension $(5 ; 2,2 ; 1,1,1,1 ; 2)$, provided that

$$
\begin{gathered}
5 \gamma+2 \beta_{2}>\delta+\alpha_{1}+\alpha_{2}+3 \beta_{1}+3 \beta_{3}+3 \beta_{4}, \gamma>\alpha_{1}+\beta_{4}, \\
\alpha_{2}+\beta_{1}>\gamma, \gamma>\alpha_{2}+\beta_{2}, 5 \gamma+2 \beta_{3}+2 \beta_{4}>\delta+\alpha_{1}+\alpha_{2}+3 \beta_{1}+3 \beta_{2}, \\
\alpha_{1}+\beta_{3}>\gamma, 5 \gamma+\beta_{1}+\beta_{2}+\beta_{4}>3 \delta+3 \alpha_{1}+3 \alpha_{2}+4 \beta_{3}, \\
\gamma=\frac{1}{5}\left(2 \delta+2 \alpha_{1}+2 \alpha_{2}+\beta_{1}+\beta_{2}+\beta_{3}+\beta_{4}\right) .
\end{gathered}
$$

In each of the above cases the representation is unique up to unitary equivalence.

Remark. Inequalities in Theorem 2- 5 can be obtained alternatively using Horn's inequalities (see, for example [3]) and W. Crawley-Boevey solution of the DeligneSimpson problem (see [1]). More precisely, representations of $\mathcal{P}_{\alpha, \beta, \delta, \gamma}$ (not necessarily *-preserving) in a fixed generalized dimension $d=(n ; \ldots)$ are in one-to-one correspondence with $n \times n$ matrices $A_{1}, A_{2}, A_{3}$ picked from fixed diagonal conjugacy classes $C_{1}, C_{2}, C_{3}$. These classes are determined by $(\alpha, \beta, \gamma, \delta)$ and $d$. Thus an application of [1. Theorem 1] to diagonal conjugacy classes gives a description of the parameters $(\alpha, \beta, \gamma, \delta)$ for which there is an irreducible (not necessarily $*$-preserving) representation of algebra $\mathcal{P}_{\alpha, \beta, \delta, \gamma}$ in terms of the root system of a certain Kac-Moody Lie algebra. In particular, it follows that there are only finitely many such representations in the case of a Dynkin graph and generalized dimensions $d$ are some simple roots (up to a certain linear transformation ([1, Theorem 1]). Moreover, for a fixed generalized dimension $d$ such a representation $\pi$ is unique. If we require that the parameters of the algebra satisfy Horn's inequalities (we must translate the parameters $\alpha, \beta, \gamma, \delta$ and the generalized dimension $d$ into eigenvalues and their multiplicities first) then $\pi$ must be unitarizable and thus $(\alpha, \beta, \gamma, \delta) \in W_{i r r}$. Thus combining Horn's inequalities and Crawley-Boevey conditions of irreducibility for $d$ we obtain inequalities in Theorem 2 5. However, to derive our results we do not use neither Horn's inequalities nor Crawley-Boevey conditions. Moreover, we give an algorithm, the Coxeter functors for $*$-representations, to construct $*$-representations of $\mathcal{P}_{\alpha, \beta, \delta, \gamma}$.

\section{References}

[1] W. Crawley-Boevey, On matrices in prescribed conjugacy classes with no common invariant subspace and sum zero Duke Math. J. -118, - N 2 (2003), p. 339-352 
[2] W. Crawley-Boevey and Ch. Geiss, Horn's problem and semi-stability for quiver representations, Representations of Algebars.-V.I. Poc. IX Intern. Conf. Benjing (2000) Red. T.Happel, V.B. Zhong Benjing, 2002, p.40-48

[3] W.Fulton, Eigenvalues, invariant factors, highest weights and Schubert calculus, Bull. Amer. Math. Soc., 37 (2000), 209-249

[4] A. A. Klyachko, Stable bundles, representation theory and Hermitian operators Sel. Math. , New ser., 4 (1998) $419-445$

[5] S. A. Kruglyak, S. V. Popovich, and Yu. S. Samořlenko. Representations of *-algebras associated with Dynkin diagrams. Uchenye Zapiski Tavricheskogo Natsional'nogo Universiteta, 16(2) (2003) 132-139, . (Russian).

[6] S. A. Kruglyak and A. V. Roiter. Locally scalar representations of graphs in the category of Hilbert spaces. Funct. Anal. and its Appl., 38(3), 2004. (Russian)

[7] S.V. Popovich, Yu.S. Samoilenko, On homomorphisms of algberas generated by projectors and Coxeter functors, Ukr. Math. Journ., 55(9) (2003) 1224-1237

[8] M. S. Vlasenko, A. C. Mellit, and Yu. S. Samořlenko. On algebras generated by linearly dependent generators that have given spectra. Funct. Anal. and its Appl., 38(4) (2004) (Russian).

[9] M.Zavodovskii, Yu. Samoilenko, *-Representations of algebras associated with Dynkin graphs, Methodth of Func. Anal. and Topology, no. 1 (2005) 